\title{
Are interventions for accelerating orthodontic tooth movement effective?
}

\author{
Abstracted from \\ Long H, Pyakurel U, Wang Y, Liao L, Zhou Y, Lai W. \\ Interventions for accelerating orthodontic tooth movement: a systematic review. \\ Angle Orthod 2013; 83: 164-171. Epub 2012/06/23. doi: 10.2319/031512-224.1. PubMed PMID: 22720793. \\ Address for correspondence: Dr Wenli Lai, State Key Laboratory of Oral Diseases, \\ Department of Orthodontics, West China School of Stomatology, Sichuan University, \\ No. 14, Section 3, Ren Min Nan Road, Chengdu, Sichuan 610041, the People's Republic of China. \\ E-mail: wenlilai@hotmail.com.
}

\section{Question: In patients undergoing orthodontic treatment, can adjunctive interventions accelerate orthodontic tooth movement compared to conventional orthodontic treatment?}

Data sources Pubmed, Embase, Sciences Citation Index, Cochrane Central Register of Controlled Trials (CENTRAL) and grey literature database of SIGLE were searched from January 1, 1990 to August 20, 2011 with no language restrictions.

Study selection Randomised controlled trials (RCTs) or quasi-RCTs in which the participants were healthy and received additional interventions to conventional orthodontic treatment for accelerating tooth movements were included. Subjects with defects in oral and maxillofacial regions (ie, cleft lip/palate), dental pathologies and medical conditions were excluded.

Data extraction and synthesis Studies were selected by two independent reviewers and disagreements were resolved by discussion with a third reviewer. The primary outcomes included accumulative moved distance (AMD) or movement rate (MR) and time required to move the tooth to its destination. Secondary outcomes were pain improvement, anchorage loss, periodontal health, orthodontic caries, pulp vitality and root resorption. The reviewers performed statistical pooling, where possible, according to a priori criteria on the basis of comparability of patient type, treatments and outcomes measured and risk of bias. The reviewers tested for heterogeneity, publication bias and sensitivity. A quality assessment test was conducted to evaluate the method used to measure AMD.

Results The authors selected seven RCTs and two quasi-RCTs, which included a total of 101 patients with an age range of 12-26.3 years.

Eight studies compared four intervention methods to no intervention group (control group). From them, four studies assessed low laser therapy (LLL), two evaluated corticotomy (CC), one assessed electrical current therapy (EC) and one evaluated pulsed electromagnetic field (PEF). Another study compared dentoalveolar distraction (DAD) vs periodontal distraction (PDD).

Quality assessment scores showed that only two studies were of high quality, five studies were of medium quality, while two studies were of low quality. All studies, except one, compared left and right sides of the same participant (split mouth design), and measured the AMD.

The method for measuring AMD was reliable in three studies, relatively reliable in one study and unreliable in four studies. The authors only performed pooled AMD mean meta-analysis for the LLL studies. The meta-analysis showed pooled mean AMD of 0.32 (95\% confidence interval $(\mathrm{Cl}), 20.04,0.68), 0.76(95 \% \mathrm{Cl}, 20.14,1.65)$, and $0.73(95 \% \mathrm{Cl}, 20.68,2.14)$ for one month, two months and three months, respectively. Two LLL studies showed no differences regarding periodontal health and two LLL studies showed no differences in root resorption between LLL intervention and control groups. Compared to control group, one study reported that CS had significantly higher MR and another study showed that CS exhibited larger AMD for one month, two months, three months and four months. Two studies revealed that CS did not show any difference in the periodontal health status. One study reported the EC showed significantly larger AMD for one month, whereas another study reported that PEF induced larger AMD for five \pm 0.6 months. DAD showed faster MR and less anchorage loss compared to PDD. Teeth remained vital in both DAD and PDD interventions and one out of six cases presented root resorption in the PDD group. Conclusions Among the five interventions corticotomy is effective and safe to accelerate orthodontic tooth movement, low-level laser therapy was unable to accelerate orthodontic tooth movement. The level of evidence does not support whether electrical current and pulsed electromagnetic fields are effective in accelerating orthodontic tooth movement and dentoalveolar or periodontal distraction is promising in accelerating orthodontic tooth movement.

\section{Commentary}

Most conventional orthodontic treatments require almost two years to complete. ${ }^{1}$ One of the aims of orthodontic treatment is to effectively treat malocclusions over the shortest duration with minimal biological side effects. Interventions to accelerate orthodontic tooth movements have been used to reduce the duration of treatment time and decrease the undesirable effects associated with long orthodontic treatments (ie root resorption, periodontitis). ${ }^{2}$ Reducing orthodontic treatment time may increase the patient's satisfaction and decrease the treatment cost. ${ }^{3,4}$ The objective of this article was to systematically review the literature that compared the effectiveness of auxiliary interventions in order to decrease the duration of orthodontic treatment with minimal adverse side effects.

This systematic review was comprehensive and well conducted with few limitations. The authors did not report any attempts to hand search related journals or retrieve data from the original authors for one of the excluded studies. In addition, the inclusion of quasi-randomised clinical trials may introduce some bias (internal validity). Furthermore, the authors did not clearly state the treatment duration, length of the follow up period or number 
of drop-outs. Even though heterogeneity, publication bias and sensitivity analysis tests were performed, the authors did not report any blinding during the selection process.

The results of this systematic review must be interpreted with caution because of the small number of high quality studies. The quality of the nine studies included was: two high, five medium and two low. Eight studies measured the accumulative moved distance (AMD) of which three were reliable, one was partially reliable and four were unreliable. One of the conclusions was that low lower laser therapy (LLL) was unable to accelerate orthodontic movement. This conclusion was based on four studies, out of which three studies were of medium quality and used unreliable methods to measure AMD. Another suggested conclusion was that corticotomy (CC) is an effective and safe method to accelerate orthodontic tooth movement based on two high quality RCT studies with reliable methods to measure AMD. However, the data from these two studies were not comparable and no meta-analysis was performed. More high quality studies assessing LLL and CC are required before reaching such conclusions with certainty. Studies assessing the other types of interventions used unreliable methodology and no conclusions regarding their effectiveness could be reached.

One important aspect that was not evaluated by any of the included studies is the cost-effectiveness of such interventions. Some of these interventions might be effective in accelerating orthodontic movement, but is the time difference justifiable when considering the additional cost? Cost-effectiveness analyses should be conducted in conjunction with the clinical trials. This will help clinicians and patients to consider the economic vs clinical impact when making informed decisions.

This review points out the limited high quality evidence in the literature. It is important to conduct future clinical research with

\section{Practice points}

- The limited evidence suggests that corticotomy may safely speed up the orthodontic tooth movement; however, its costeffectiveness needs to be further assessed.

- Evidence is inconclusive for the effectiveness of other interventional techniques. Future high quality RCTs considering the additional cost of such interventions are necessary to help dental practitioners make informed clinical decisions.

high standards, so that clinicians can comfortably make their clinical decisions depending on sound scientific bases.

Mohamed-Nur Abdallah' ${ }^{1}$ and Carlos Flores-Mir ${ }^{2}$

${ }^{1}$ Faculty of Dentistry, McGill University, Quebec, Canada

${ }^{2}$ Division of Orthodontics, Department of Dentistry, Faculty of Medicine and Dentistry, University of Alberta, Edmonton, Canada

1. Skidmore KJ, Brook KJ, Thomson WM, Harding WJ. Factors influencing treatment time in orthodontic patients. Am / Orthod Dentofacial Orthop 2006; 129: 230-238.

2. Nimeri G, Kau CH, Abou-Kheir NS, Corona R. Acceleration of tooth movement during orthodontic treatment - a frontier in orthodontics. Prog Orthod 2013; 14: 42.

3. Riedmann T, Georg T, Berg R. Adult patients' view of orthodontic treatment outcome compared to professional assessments. J Orofac Orthop 1999; 60: 308-320.

4. Mavreas D, Athanasiou AE. Factors affecting the duration of orthodontic treatment: a systematic review. Eur J Orthod 2008; 30: 386-395.

\section{Editors note:}

Since this article was submitted another review on this topic has been published, see pages 114-115 of this issue.

1. Gkantidis N, Mistakidis I, Kouskoura T, Pandis N. Effectiveness of non-conventional methods for accelerated orthodontic tooth movement: a systematic review and meta-analysis. J Dent 2014; 42: 1300-1319. pii: S0300-5712(14)00218-8. doi: 10.1016/j.jdent.2014.07.013. [Epub ahead of print] PubMed PMID: 25072362

Evidence-Based Dentistry (2014) 15, 116-117. doi:10.1038/sj.ebd.6401065 